BABAR-CONF-03/25

SLAC-PUB-10104

hep-ex/0308021

August 2003

\title{
Measurement of the $B^{0} \rightarrow K_{2}^{*}(1430)^{0} \gamma$ and $B^{+} \rightarrow K_{2}^{*}(1430)^{+} \gamma$ branching fractions
}

\author{
The BABAR Collaboration
}

August 8, 2003

\begin{abstract}
We have investigated the exclusive, radiative $B$-meson decay to the charmless meson $K_{2}^{*}(1430)$ in $88.5 \times 10^{6} B \bar{B}$ events accumulated between 1999 and 2002 with the BABAR detector at the PEP-II storage ring. We present a preliminary measurement of the branching fractions $\mathcal{B}\left(B^{0} \rightarrow\right.$ $\left.K_{2}^{*}(1430)^{0} \gamma\right)=(1.22 \pm 0.25 \pm 0.11) \times 10^{-5}$ and $\mathcal{B}\left(B^{+} \rightarrow K_{2}^{*}(1430)^{+} \gamma\right)=(1.44 \pm 0.40 \pm 0.13) \times 10^{-5}$, where the first error is statistical and the second systematic.
\end{abstract}

Contributed to the $\mathrm{XXI}^{\mathrm{st}}$ International Symposium on Lepton and Photon Interactions at High Energies, 8/11 - 8/16/2003, Fermilab, Illinois USA

Stanford Linear Accelerator Center, Stanford University, Stanford, CA 94309

Work supported in part by Department of Energy contract DE-AC03-76SF00515. 
The BABAR Collaboration,

B. Aubert, R. Barate, D. Boutigny, J.-M. Gaillard, A. Hicheur, Y. Karyotakis, J. P. Lees, P. Robbe, V. Tisserand, A. Zghiche

Laboratoire de Physique des Particules, F-74941 Annecy-le-Vieux, France

A. Palano, A. Pompili

Università di Bari, Dipartimento di Fisica and INFN, I-70126 Bari, Italy

J. C. Chen, N. D. Qi, G. Rong, P. Wang, Y. S. Zhu

Institute of High Energy Physics, Beijing 100039, China

G. Eigen, I. Ofte, B. Stugu

University of Bergen, Inst. of Physics, N-5007 Bergen, Norway

G. S. Abrams, A. W. Borgland, A. B. Breon, D. N. Brown, J. Button-Shafer, R. N. Cahn, E. Charles, C. T. Day, M. S. Gill, A. V. Gritsan, Y. Groysman, R. G. Jacobsen, R. W. Kadel, J. Kadyk, L. T. Kerth, Yu. G. Kolomensky, J. F. Kral, G. Kukartsev, C. LeClerc, M. E. Levi, G. Lynch, L. M. Mir, P. J. Oddone,

T. J. Orimoto, M. Pripstein, N. A. Roe, A. Romosan, M. T. Ronan, V. G. Shelkov, A. V. Telnov, W. A. Wenzel

Lawrence Berkeley National Laboratory and University of California, Berkeley, CA 94720, USA

K. Ford, T. J. Harrison, C. M. Hawkes, D. J. Knowles, S. E. Morgan, R. C. Penny, A. T. Watson, N. K. Watson

University of Birmingham, Birmingham, B15 2TT, United Kingdom

T. Held, K. Goetzen, H. Koch, B. Lewandowski, M. Pelizaeus, K. Peters, H. Schmuecker, M. Steinke Ruhr Universität Bochum, Institut für Experimentalphysik 1, D-44780 Bochum, Germany

N. R. Barlow, J. T. Boyd, N. Chevalier, W. N. Cottingham, M. P. Kelly, T. E. Latham, C. Mackay, F. F. Wilson

University of Bristol, Bristol BS8 1TL, United Kingdom

K. Abe, T. Cuhadar-Donszelmann, C. Hearty, T. S. Mattison, J. A. McKenna, D. Thiessen

University of British Columbia, Vancouver, BC, Canada V6T $1 Z 1$

P. Kyberd, A. K. McKemey

Brunel University, Uxbridge, Middlesex UB8 3PH, United Kingdom

V. E. Blinov, A. D. Bukin, V. B. Golubev, V. N. Ivanchenko, E. A. Kravchenko, A. P. Onuchin,

S. I. Serednyakov, Yu. I. Skovpen, E. P. Solodov, A. N. Yushkov

Budker Institute of Nuclear Physics, Novosibirsk 630090, Russia

D. Best, M. Bruinsma, M. Chao, D. Kirkby, A. J. Lankford, M. Mandelkern, R. K. Mommsen, W. Roethel, D. P. Stoker

University of California at Irvine, Irvine, CA 92697, USA

C. Buchanan, B. L. Hartfiel

University of California at Los Angeles, Los Angeles, CA 90024, USA 
B. C. Shen

University of California at Riverside, Riverside, CA 92521, USA

D. del Re, H. K. Hadavand, E. J. Hill, D. B. MacFarlane, H. P. Paar, Sh. Rahatlou, V. Sharma

University of California at San Diego, La Jolla, CA 92093, USA

J. W. Berryhill, C. Campagnari, B. Dahmes, N. Kuznetsova, S. L. Levy, O. Long, A. Lu, M. A. Mazur, J. D. Richman, W. Verkerke

University of California at Santa Barbara, Santa Barbara, CA 93106, USA

T. W. Beck, J. Beringer, A. M. Eisner, C. A. Heusch, W. S. Lockman, T. Schalk, R. E. Schmitz, B. A. Schumm, A. Seiden, M. Turri, W. Walkowiak, D. C. Williams, M. G. Wilson

University of California at Santa Cruz, Institute for Particle Physics, Santa Cruz, CA 95064, USA

J. Albert, E. Chen, G. P. Dubois-Felsmann, A. Dvoretskii, D. G. Hitlin, I. Narsky, F. C. Porter, A. Ryd, A. Samuel, S. Yang

California Institute of Technology, Pasadena, CA 91125, USA

S. Jayatilleke, G. Mancinelli, B. T. Meadows, M. D. Sokoloff

University of Cincinnati, Cincinnati, $\mathrm{OH}$ 45221, USA

T. Abe, F. Blanc, P. Bloom, S. Chen, P. J. Clark, W. T. Ford, U. Nauenberg, A. Olivas, P. Rankin, J. Roy, J. G. Smith, W. C. van Hoek, L. Zhang University of Colorado, Boulder, CO 80309, USA

J. L. Harton, T. Hu, A. Soffer, W. H. Toki, R. J. Wilson, J. Zhang

Colorado State University, Fort Collins, CO 80523, USA

D. Altenburg, T. Brandt, J. Brose, T. Colberg, M. Dickopp, R. S. Dubitzky, A. Hauke, H. M. Lacker, E. Maly, R. Müller-Pfefferkorn, R. Nogowski, S. Otto, J. Schubert, K. R. Schubert, R. Schwierz, B. Spaan, L. Wilden

Technische Universität Dresden, Institut für Kern- und Teilchenphysik, D-01062 Dresden, Germany

D. Bernard, G. R. Bonneaud, F. Brochard, J. Cohen-Tanugi, P. Grenier, Ch. Thiebaux, G. Vasileiadis, M. Verderi

Ecole Polytechnique, LLR, F-91128 Palaiseau, France

A. Khan, D. Lavin, F. Muheim, S. Playfer, J. E. Swain

University of Edinburgh, Edinburgh EH9 3JZ, United Kingdom

M. Andreotti, V. Azzolini, D. Bettoni, C. Bozzi, R. Calabrese, G. Cibinetto, E. Luppi, M. Negrini, L. Piemontese, A. Sarti

Università di Ferrara, Dipartimento di Fisica and INFN, I-44100 Ferrara, Italy

E. Treadwell

Florida AEM University, Tallahassee, FL 3230\%, USA

F. Anulli, ${ }^{1}$ R. Baldini-Ferroli, M. Biasini, ${ }^{1}$ A. Calcaterra, R. de Sangro, D. Falciai, G. Finocchiaro, P. Patteri, I. M. Peruzzi, ${ }^{1}$ M. Piccolo, M. Pioppi, ${ }^{1}$ A. Zallo

Laboratori Nazionali di Frascati dell'INFN, I-00044 Frascati, Italy

\footnotetext{
${ }^{1}$ Also with Università di Perugia, Perugia, Italy
} 
A. Buzzo, R. Capra, R. Contri, G. Crosetti, M. Lo Vetere, M. Macri, M. R. Monge, S. Passaggio, C. Patrignani, E. Robutti, A. Santroni, S. Tosi Università di Genova, Dipartimento di Fisica and INFN, I-16146 Genova, Italy

S. Bailey, M. Morii, E. Won

Harvard University, Cambridge, MA 02138, USA

W. Bhimji, D. A. Bowerman, P. D. Dauncey, U. Egede, I. Eschrich, J. R. Gaillard, G. W. Morton, J. A. Nash, P. Sanders, G. P. Taylor Imperial College London, London, SW7 2BW, United Kingdom

G. J. Grenier, S.-J. Lee, U. Mallik

University of Iowa, Iowa City, IA 52242, USA

J. Cochran, H. B. Crawley, J. Lamsa, W. T. Meyer, S. Prell, E. I. Rosenberg, J. Yi

Iowa State University, Ames, IA 50011-3160, USA

M. Davier, G. Grosdidier, A. Höcker, S. Laplace, F. Le Diberder, V. Lepeltier, A. M. Lutz, T. C. Petersen, S. Plaszczynski, M. H. Schune, L. Tantot, G. Wormser Laboratoire de l'Accélérateur Linéaire, F-91898 Orsay, France

V. Brigljević , C. H. Cheng, D. J. Lange, D. M. Wright Lawrence Livermore National Laboratory, Livermore, CA 94550, USA

A. J. Bevan, J. P. Coleman, J. R. Fry, E. Gabathuler, R. Gamet, M. Kay, R. J. Parry, D. J. Payne, R. J. Sloane, C. Touramanis University of Liverpool, Liverpool L69 3BX, United Kingdom

J. J. Back, P. F. Harrison, H. W. Shorthouse, P. Strother, P. B. Vidal Queen Mary, University of London, E1 4NS, United Kingdom

C. L. Brown, G. Cowan, R. L. Flack, H. U. Flaecher, S. George, M. G. Green, A. Kurup, C. E. Marker, T. R. McMahon, S. Ricciardi, F. Salvatore, G. Vaitsas, M. A. Winter

University of London, Royal Holloway and Bedford New College, Egham, Surrey TW20 0EX, United Kingdom

D. Brown, C. L. Davis

University of Louisville, Louisville, KY 40292, USA

J. Allison, R. J. Barlow, A. C. Forti, P. A. Hart, M. C. Hodgkinson, F. Jackson, G. D. Lafferty, A. J. Lyon, J. H. Weatherall, J. C. Williams University of Manchester, Manchester M13 9PL, United Kingdom

A. Farbin, A. Jawahery, D. Kovalskyi, C. K. Lae, V. Lillard, D. A. Roberts University of Maryland, College Park, MD 20742, USA

G. Blaylock, C. Dallapiccola, K. T. Flood, S. S. Hertzbach, R. Kofler, V. B. Koptchev, T. B. Moore, S. Saremi, H. Staengle, S. Willocq

University of Massachusetts, Amherst, MA 01003, USA 
R. Cowan, G. Sciolla, F. Taylor, R. K. Yamamoto

Massachusetts Institute of Technology, Laboratory for Nuclear Science, Cambridge, MA 02139, USA

D. J. J. Mangeol, P. M. Patel

McGill University, Montréal, QC, Canada H3A 2T8

A. Lazzaro, F. Palombo

Università di Milano, Dipartimento di Fisica and INFN, I-20133 Milano, Italy

J. M. Bauer, L. Cremaldi, V. Eschenburg, R. Godang, R. Kroeger, J. Reidy, D. A. Sanders, D. J. Summers, H. W. Zhao

University of Mississippi, University, MS 38677, USA

S. Brunet, D. Cote-Ahern, C. Hast, P. Taras

Université de Montréal, Laboratoire René J. A. Lévesque, Montréal, QC, Canada H3C 3J7

H. Nicholson

Mount Holyoke College, South Hadley, MA 01075, USA

C. Cartaro, N. Cavallo, ${ }^{2}$ G. De Nardo, F. Fabozzi, ${ }^{2}$ C. Gatto, L. Lista, P. Paolucci, D. Piccolo, C. Sciacca Università di Napoli Federico II, Dipartimento di Scienze Fisiche and INFN, I-80126, Napoli, Italy

M. A. Baak, G. Raven

NIKHEF, National Institute for Nuclear Physics and High Energy Physics, NL-1009 DB Amsterdam, The Netherlands

J. M. LoSecco

University of Notre Dame, Notre Dame, IN 46556, USA

T. A. Gabriel

Oak Ridge National Laboratory, Oak Ridge, TN 37831, USA

B. Brau, K. K. Gan, K. Honscheid, D. Hufnagel, H. Kagan, R. Kass, T. Pulliam, Q. K. Wong

Ohio State University, Columbus, OH 43210, USA

J. Brau, R. Frey, C. T. Potter, N. B. Sinev, D. Strom, E. Torrence

University of Oregon, Eugene, OR 97403, USA

F. Colecchia, A. Dorigo, F. Galeazzi, M. Margoni, M. Morandin, M. Posocco, M. Rotondo, F. Simonetto, R. Stroili, G. Tiozzo, C. Voci

Università di Padova, Dipartimento di Fisica and INFN, I-35131 Padova, Italy

M. Benayoun, H. Briand, J. Chauveau, P. David, Ch. de la Vaissière, L. Del Buono, O. Hamon, M. J. J. John, Ph. Leruste, J. Ocariz, M. Pivk, L. Roos, J. Stark, S. T'Jampens, G. Therin

Universités Paris VI et VII, Lab de Physique Nucléaire H. E., F-75252 Paris, France

P. F. Manfredi, V. Re

Università di Pavia, Dipartimento di Elettronica and INFN, I-27100 Pavia, Italy

\footnotetext{
${ }^{2}$ Also with Università della Basilicata, Potenza, Italy
} 


\author{
P. K. Behera, L. Gladney, Q. H. Guo, J. Panetta \\ University of Pennsylvania, Philadelphia, PA 19104, USA
}

C. Angelini, G. Batignani, S. Bettarini, M. Bondioli, F. Bucci, G. Calderini, M. Carpinelli, V. Del Gamba, F. Forti, M. A. Giorgi, A. Lusiani, G. Marchiori, F. Martinez-Vidal, ${ }^{3}$ M. Morganti, N. Neri, E. Paoloni, M. Rama, G. Rizzo, F. Sandrelli, J. Walsh

Università di Pisa, Dipartimento di Fisica, Scuola Normale Superiore and INFN, I-56127 Pisa, Italy

M. Haire, D. Judd, K. Paick, D. E. Wagoner

Prairie View A\&M University, Prairie View, TX 77446, USA

N. Danielson, P. Elmer, C. Lu, V. Miftakov, J. Olsen, A. J. S. Smith, H. A. Tanaka E. W. Varnes Princeton University, Princeton, NJ 08544, USA

F. Bellini, G. Cavoto, ${ }^{4}$ R. Faccini, ${ }^{5}$ F. Ferrarotto, F. Ferroni, M. Gaspero, M. A. Mazzoni, S. Morganti, M. Pierini, G. Piredda, F. Safai Tehrani, C. Voena

Università di Roma La Sapienza, Dipartimento di Fisica and INFN, I-00185 Roma, Italy

S. Christ, G. Wagner, R. Waldi

Universität Rostock, D-18051 Rostock, Germany

T. Adye, N. De Groot, B. Franek, N. I. Geddes, G. P. Gopal, E. O. Olaiya, S. M. Xella

Rutherford Appleton Laboratory, Chilton, Didcot, Oxon, OX11 0QX, United Kingdom

R. Aleksan, S. Emery, A. Gaidot, S. F. Ganzhur, P.-F. Giraud, G. Hamel de Monchenault, W. Kozanecki, M. Langer, M. Legendre, G. W. London, B. Mayer, G. Schott, G. Vasseur, Ch. Yeche, M. Zito

DSM/Dapnia, CEA/Saclay, F-91191 Gif-sur-Yvette, France

M. V. Purohit, A. W. Weidemann, F. X. Yumiceva

University of South Carolina, Columbia, SC 29208, USA

D. Aston, R. Bartoldus, N. Berger, A. M. Boyarski, O. L. Buchmueller, M. R. Convery, D. P. Coupal, D. Dong, J. Dorfan, D. Dujmic, W. Dunwoodie, R. C. Field, T. Glanzman, S. J. Gowdy, E. Grauges-Pous,

T. Hadig, V. Halyo, T. Hryn'ova, W. R. Innes, C. P. Jessop, M. H. Kelsey, P. Kim, M. L. Kocian, U. Langenegger, D. W. G. S. Leith, S. Luitz, V. Luth, H. L. Lynch, H. Marsiske, R. Messner, D. R. Muller, C. P. O'Grady, V. E. Ozcan, A. Perazzo, M. Perl, S. Petrak, B. N. Ratcliff, S. H. Robertson, A. Roodman,

A. A. Salnikov, R. H. Schindler, J. Schwiening, G. Simi, A. Snyder, A. Soha, J. Stelzer, D. Su,

M. K. Sullivan, J. Va'vra, S. R. Wagner, M. Weaver, A. J. R. Weinstein, W. J. Wisniewski, D. H. Wright, C. C. Young

Stanford Linear Accelerator Center, Stanford, CA 94309, USA

P. R. Burchat, A. J. Edwards, T. I. Meyer, B. A. Petersen, C. Roat

Stanford University, Stanford, CA 94305-4060, USA

S. Ahmed, M. S. Alam, J. A. Ernst, M. Saleem, F. R. Wappler

State Univ. of New York, Albany, NY 12222, USA

\footnotetext{
${ }^{3}$ Also with IFIC, Instituto de Física Corpuscular, CSIC-Universidad de Valencia, Valencia, Spain

${ }^{4}$ Also with Princeton University

${ }^{5}$ Also with University of California at San Diego
} 
W. Bugg, M. Krishnamurthy, S. M. Spanier

University of Tennessee, Knoxville, TN 37996, USA

R. Eckmann, H. Kim, J. L. Ritchie, R. F. Schwitters

University of Texas at Austin, Austin, TX 78712, USA

J. M. Izen, I. Kitayama, X. C. Lou, S. Ye

University of Texas at Dallas, Richardson, TX 75083, USA

F. Bianchi, M. Bona, F. Gallo, D. Gamba

Università di Torino, Dipartimento di Fisica Sperimentale and INFN, I-10125 Torino, Italy

C. Borean, L. Bosisio, G. Della Ricca, S. Dittongo, S. Grancagnolo, L. Lanceri, P. Poropat, ${ }^{6}$ L. Vitale, G. Vuagnin

Università di Trieste, Dipartimento di Fisica and INFN, I-34127 Trieste, Italy

R. S. Panvini

Vanderbilt University, Nashville, TN 37235, USA

Sw. Banerjee, C. M. Brown, D. Fortin, P. D. Jackson, R. Kowalewski, J. M. Roney

University of Victoria, Victoria, BC, Canada V8W $3 P 6$

H. R. Band, S. Dasu, M. Datta, A. M. Eichenbaum, J. R. Johnson, P. E. Kutter, H. Li, R. Liu, F. Di Lodovico, A. Mihalyi, A. K. Mohapatra, Y. Pan, R. Prepost, S. J. Sekula, J. H. von Wimmersperg-Toeller, J. Wu, S. L. Wu, Z. Yu

University of Wisconsin, Madison, WI 53706, USA

H. Neal

Yale University, New Haven, CT 06511, USA

\footnotetext{
${ }^{6}$ Deceased
} 


\section{INTRODUCTION}

In the Standard Model, flavor-changing neutral currents (FCNC) are forbidden at the tree level. For example, there is no direct coupling between the $b$ quark and the $s$ or $d$ quarks. Effective FCNC are induced by loop (or "penguin") diagrams, where a quark emits and re-absorbs a $W$ thus changing flavor twice, such as the $b \rightarrow s \gamma$ transition depicted in Fig. 1.

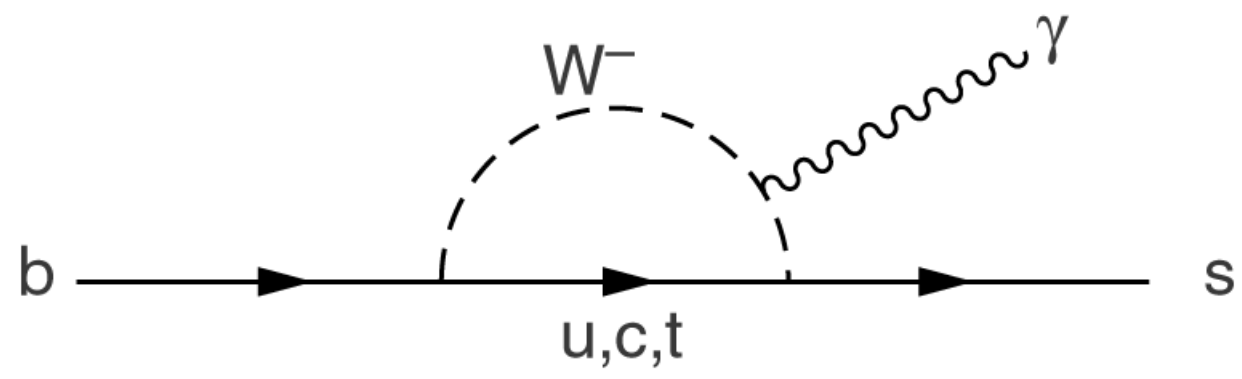

Figure 1: $b \rightarrow s \gamma$ "penguin" diagram.

With large samples of $B$-mesons and increasingly powerful background-suppression techniques, experimenters have succeeded in measuring Cabibbo-Kobayashi-Mashawa favored penguins. Theory has also made good progress in computing the decay rates; in particular, recent completion of the NLO correction [1] to the inclusive $b \rightarrow s \gamma$ decay rate has sparked experimental efforts to further improve the measurement. Comparisons between theoretical and experimental rates place strong constraints on physics beyond the Standard Model [2].

The discovery of $B \rightarrow K^{*}(892) \gamma$ by the CLEO collaboration [3] verified the existence of penguins. The same publication also reported the evidence for $B \rightarrow K_{2}^{*}(1430) \gamma$, later confirmed by the BELLE collaboration [4]. Measurements of the inclusive decay rate have been made by several experiments [5]. Detailed knowledge about the decays to resonant modes with masses higher than $K^{*}(892)$, such as $B \rightarrow K_{2}^{*}(1430) \gamma$ decay, will provide a better understanding of the inclusive $b \rightarrow s \gamma$ branching fraction in terms of the sum over exclusive modes.

\section{THE BABAR DETECTOR AND DATASET}

This study is based on $81.4 \mathrm{fb}^{-1}$ of data collected at the $\Upsilon(4 S)$ resonance ("on-resonance") with the BABAR detector at the PEP-II asymmetric $e^{+}(3.1 \mathrm{GeV})-e^{-}(9.0 \mathrm{GeV})$ storage ring, corresponding to $88.5 \times 10^{6} B \bar{B}$ pairs. We have also collected a data sample of $9.6 \mathrm{fb}^{-1}$ at $40 \mathrm{MeV}$ below the $\Upsilon(4 S)$ energy ("off-resonance"). The number of $B \bar{B}$ meson pairs is determined from the ratio of the number of hadronic events to muon pairs in on- and off-resonance data. We assume that the $\Upsilon(4 S)$ decays equally to neutral and charged $B$-meson pairs.

The BABAR detector is described elsewhere [6]. The analysis described below makes use of charged track, $K_{S}$ and $\pi^{0}$ reconstruction, along with the charged particle identification. Charged particle trajectories are measured by a 5-layer double-sided silicon vertex tracker (SVT) and a 40-layer drift chamber, which also provide ionization measurements $(d E / d x)$ used for particle identification. For charged tracks with momentum $p>1 \mathrm{GeV} / c$, the measured transverse momentum 
with respect to the beam axis $\left(p_{T}\right)$ has the resolution

$$
\frac{\sigma_{p_{T}}}{p_{T}}=0.13 \% p_{T}+0.45 \%
$$

where $p_{T}$ is measured in $\mathrm{GeV} / c$.

Photons and electrons are measured in the barrel and forward end-cap electromagnetic calorimeter, consisting of 6580 Thallium-doped CsI crystals. The electromagnetic calorimeter resolution, $\sigma_{E}$, can be expressed as

$$
\frac{\sigma_{E}}{E}=\frac{2.3 \%}{E^{\frac{1}{4}}} \oplus 1.9 \%
$$

where the energy $E$ is measured in $\mathrm{GeV}$.

Charged particle identification is provided by the energy loss $(d E / d x)$ in the tracking devices and by an internally reflecting ring-imaging Cherenkov detector (DIRC). The DIRC transports the Cherenkov light to a water-filled expansion volume equipped with approximately 11,000 photomultiplier tubes. A $K / \pi$ separation better than four standard deviations is achieved for momenta below $3 \mathrm{GeV} / c$.

We use Monte Carlo simulations of the BABAR detector based on GEANT 4 [7] to optimize our selection criteria and to determine signal efficiencies. These simulations take into account the varying detector conditions and beam backgrounds during the data-taking period.

\section{B CANDIDATE RECONSTRUCTION}

The $K_{2}^{*}(1430)$ is reconstructed from $K^{+}, K^{0}, \pi^{+}$and $\pi^{0}$ candidates through the three modes $K_{2}^{* 0}(1430) \rightarrow K^{+} \pi^{-}$and $K_{2}^{*+}(1430) \rightarrow K^{+} \pi^{0}, K^{0} \pi^{+} . K^{0}$ mesons are only reconstructed from the decay $K_{S}^{0} \rightarrow \pi^{+} \pi^{-}$. In this paper the charge conjugate decays are implied unless otherwise stated.

A photon candidate is defined as a localized energy maximum within the calorimeter acceptance $-0.74<\cos \theta<0.93$, where $\theta$ is the polar angle to the detector axis. It must be isolated by $25 \mathrm{~cm}$ from any other neutral candidate or track and have a lateral energy profile consistent with a photon shower. To suppress photons from $\pi^{0}(\eta)$ decays, we veto any photon that combines with another photon of energy greater than 50 (250) $\mathrm{MeV}$ to form a $\gamma \gamma$ invariant mass in the range $115(508)<M_{\gamma \gamma}<155(588) \mathrm{MeV} / c^{2}$.

The $\pi^{0}$ candidates are reconstructed from pairs of photons, which have energy above $50 \mathrm{MeV}$ and opening angle less than 36 degrees; the invariant mass of the two photons is required to be in the range $115<M_{\gamma \gamma}<150 \mathrm{MeV} / c^{2}$; the $\pi^{0}$ momentum is recalculated with a $\pi^{0}$ mass constraint to improve the energy resolution.

The $K^{ \pm}$and $\pi^{ \pm}$track candidates are required to be well reconstructed in the drift chamber and to originate from a vertex consistent with the $e^{+} e^{-}$interaction point (IP). A track is identified as a kaon if it is projected to pass through the fiducial volume of the DIRC, and the cone of Cherenkov light is consistent in time and angle with a kaon of the measured track momentum. A charged pion is identified as a track that does not satisfy the criteria for a kaon or an electron.

The $K_{S}^{0}$ candidates are reconstructed from two oppositely charged tracks coming from a common vertex displaced from the IP by at least $0.2 \mathrm{~cm}$. Each candidate is required to lie in a direction consistent with the $K_{S}^{0}$ momentum, while having an invariant mass within a $489<M_{\pi^{+} \pi^{-}}<$ $507 \mathrm{MeV} / c^{2}$ window around the nominal $K_{S}^{0}$ mass. 
The $K_{2}^{*}(1430)$ candidate is required to have a $K \pi$ invariant mass within 120 and $110 \mathrm{MeV} / c^{2}$ of the known $K_{2}^{* 0}$ and $K_{2}^{*+}$ mass [8]. For the $K^{+} \pi^{-}$mode, we require that the two tracks originate from a common vertex with a probability of at least 0.001 .

To remove Bhabha, radiative-Bhabha and tau events, we require that the second-order FoxWolfram moment [9] of the event is less than 0.9 .

The $B$ candidates are reconstructed by combining one $K_{2}^{*}(1430)$ and one $\gamma$ candidate with energy $1.80<E_{\gamma}^{*}<2.75 \mathrm{GeV}$ in the rest frame of the $\Upsilon(4 S)$. In this frame, the energy of the $B$-meson is given by the beam energy, $\sqrt{s} / 2$, which is known much more precisely than the energy of the $B$ candidate. Therefore, to isolate the $B$-meson signal, we use two kinematic variables: the difference between the reconstructed energy of the $B$ candidate and the beam energy in the center-of-mass frame $(\Delta E)$, and the beam energy substituted mass $\left(m_{\mathrm{ES}}\right)$, defined as

$$
m_{E S}^{\mathrm{raw}}=\sqrt{E_{\text {beam }}^{2}-p_{B}^{* 2}}
$$

where

$$
\begin{aligned}
& E_{\text {beam }}=\sqrt{s} / 2, \\
& \vec{p}_{B}^{*}=\vec{p}_{K^{*}}^{*}+\vec{p}_{\gamma}^{*} .
\end{aligned}
$$

For the modes containing a single photon candidate, namely $K^{+} \pi^{-}$and $K_{S}^{0} \pi^{+}$, we adopt a technique from the CLEO analysis [3], which rescales the measured photon energy $E_{\gamma}^{*}$ with a factor $\kappa$, determined for each event, such that $E_{K^{*}}^{*}+\kappa E_{\gamma}^{*}-E_{\text {beam }}^{*}=0$; this improves the $m_{\mathrm{ES}}$ resolution from 3.0 to $2.7 \mathrm{MeV} / c^{2}$. If we find multiple candidates with $|\Delta E|<0.3 \mathrm{GeV}$ and $m_{\mathrm{ES}}>5.2 \mathrm{GeV} / c^{2}$ in the same event, which happens in $3.1 \%, 6.3 \%$ and $4.9 \%$ of the events for the $K^{+} \pi^{-}, K_{S}^{0} \pi^{+}$ and $K^{+} \pi^{0}$ modes, respectively, we take the candidate that has the invariant mass closest to the $K_{2}^{*}(1430)$ mass [8].

The background has two components, one of which is continuum $q \bar{q}$ production, where $q$ can be a $u, d, s$ or $c$ quark, with the high-energy photon originating from initial-state radiation or from $\pi^{0}$ and $\eta$ decays. (This is known as the "non peaking" background in the $m_{\mathrm{ES}}$ and $\Delta E$ distributions.)

The second background contribution is from other $B \rightarrow X_{s} \gamma$ modes (dominantly $B \rightarrow K^{*}(1410) \gamma$ ) and non resonant $B \rightarrow K \pi \gamma$ decays. We label these the "peaking" background, since these decays have $m_{\mathrm{ES}}$ and $\Delta E$ distributions similar to the signal.

In order to distinguish the $B \rightarrow K_{2}^{*}(1430) \gamma$ signal from $B \rightarrow K^{*}(1410) \gamma$ and non resonant decays, we examine the helicity angle distributions. The helicity angle $\theta_{H}$ is defined as the angle of the $K^{+}$or $K_{S}^{0}$ in the rest frame of the $K_{2}^{*}(1430)$ with respect to the flight direction of the $K_{2}^{*}(1430)$. All three modes have different helicity angle distributions: $\sin ^{2} \theta_{H} \cos ^{2} \theta_{H}$ for $K_{2}^{*}(1430), \sin ^{2} \theta_{H}$ for $K^{*}(1410)$ and primarily $\sin ^{2} \theta_{H}$ for non resonant decays assuming $J=1$ for the spin of the $K \pi$ system. The non resonant decays may have higher angular momentum contributions but the lowest possible angular momentum state is dominant, so the helicity angle distribution for non resonant decay is attributed to be the same as that of $B \rightarrow K^{*}(1410) \gamma$ decay. The systematic uncertainty related with this modeling is studied and included in the measured branching fraction uncertainty.

\section{BACKGROUND SUPPRESSION}

We have exploited the difference in the event topology between signal and continuum background to reduce the continuum contribution, as well as the combinatorial $B \bar{B}$ background. The distribution of the thrust angle, defined as the angle between the direction of the photon candidate 
and the thrust axis of the rest of the event (the particles not used in the reconstruction of the $\mathrm{B}$ candidate) in the center-of-mass frame, is shown in Fig. 2. We train a neural network with a combination of the thrust angle, the angle of the $B$-meson candidate's direction with respect to the beam axis, the center-of-mass momentum vectors for tracks and photons not associated with the $B$ candidate (binned in $10^{\circ}$ intervals ranging from parallel to anti parallel relative to the photon momentum), sphericity and the ratio of second-to-zeroth order Fox-Wolfram moments in the photon recoil system. The neural network improves background suppression significantly. The distribution of the neural network output is shown in Fig. 3 for MC signal, MC continuum background and off-resonance data. The neural network software used for this analysis is based on the Stuttgart Neural Network Simulator [10].

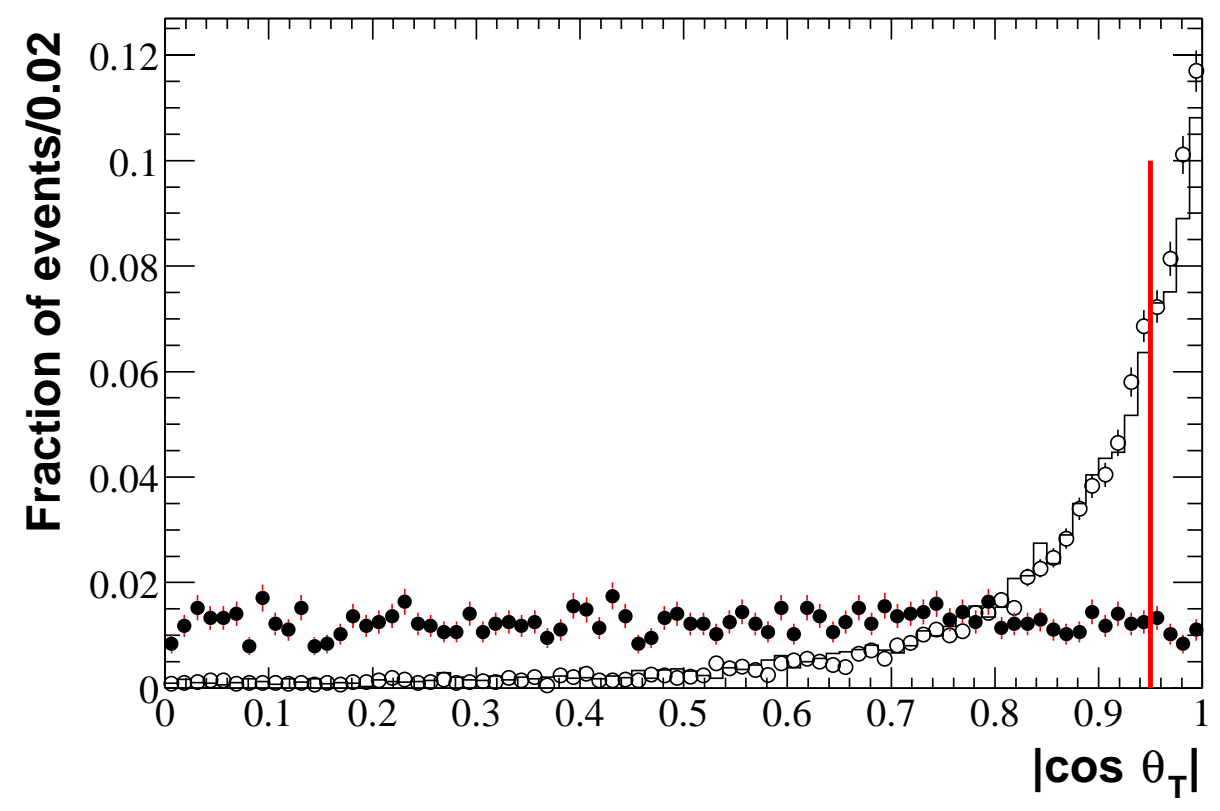

Figure 2: Thrust angle distribution: Filled circles correspond to $B \rightarrow K_{2}^{*}(1430) \gamma$ signal MC simulation, the histogram corresponds to off-resonance data, and the open circles correspond to continuum background MC simulation. The vertical line indicates the cut value.

The cuts on thrust angle $\left(\theta_{\mathrm{T}}\right)$ and neural network output (NNO) have been optimized using an iterative method to minimize correlations. The final cuts are $\left|\cos \theta_{\mathrm{T}}\right|<0.95$ and $\mathrm{NNO}>0.55$, shown in Figs. 2 and 3. Because the variables used for the neural network training are mostly calculated from the rest-of-the-event information, we use a sample of fully reconstructed $B \rightarrow D \pi^{-}$ candidates in data, as well as a sample of simulated $B \rightarrow D \pi^{-}$events as control samples. The bachelor pion in the $B \rightarrow D \pi^{-}$decay is treated like the photon in $B \rightarrow K_{2}^{*}(1430) \gamma$ decay for the calculations of the event variables; the difference in the efficiency of the cut on the neural network output between different samples is taken as the systematic uncertainty related to this cut. 


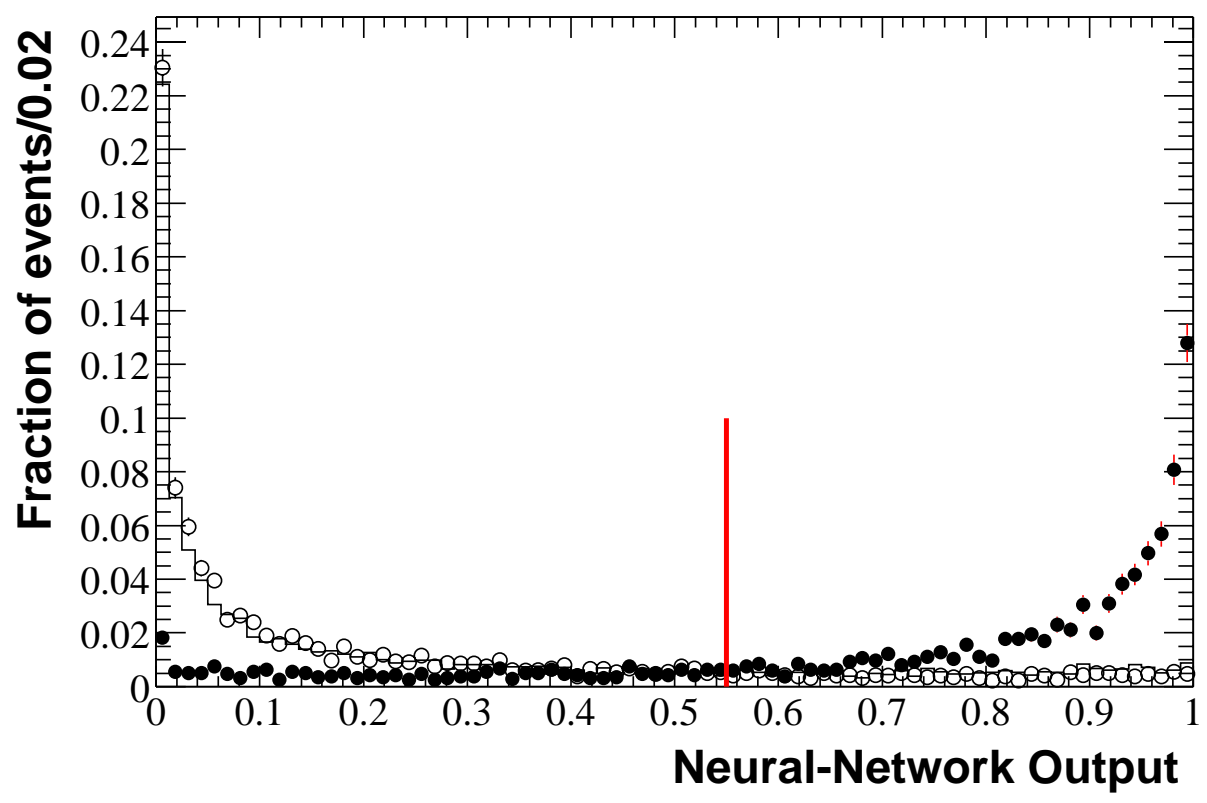

Figure 3: Neural network output distribution: Filled circles correspond to $B \rightarrow K_{2}^{*}(1430) \gamma$ signal MC simulation, the histogram corresponds to off-resonance data, and the open circles correspond to continuum background MC simulation. The vertical line indicates the cut value.

\section{ANALYSIS METHOD}

An unbinned maximum-likelihood technique is used to fit simultaneously the $m_{\mathrm{ES}}, \Delta E$ and $\left|\cos \theta_{H}\right|$ distributions. The fit is performed independently for each of the decay modes considered here.

The signal $m_{\mathrm{ES}}$ and $\Delta E$ distributions are well described by an asymmetric resolution function ("Crystal-Ball" function) [11] $S$, having an approximately Gaussian core plus a long tail due to the energy leakage from the calorimeter for the photon candidates:

$$
S \propto \begin{cases}\exp \left(-\left(m-m_{0}\right)^{2} /\left(2 \sigma^{2}\right)\right) & \text { for } m>m_{0}-\alpha \sigma, \\ \frac{(n / \alpha)^{n} \exp \left(-\alpha^{2} / 2\right)}{\left(\left(m_{0}-m\right) / \sigma+n / \alpha-\alpha\right)^{n}} & \text { for } m \leqslant m_{0}-\alpha \sigma .\end{cases}
$$

The continuum background is parameterized empirically by a threshold function [12] for $m_{\mathrm{ES}}$ and a linear function for $\Delta E$.

The $\cos \theta_{H}$ distribution of the signal has been parameterized with $\sin ^{2} \theta_{H} \cos ^{2} \theta_{H}-\lambda\left(\cos ^{4} \theta_{H}-\right.$ $\cos ^{6} \theta_{H}$ ), where $\lambda$ is a parameter determined from the Monte Carlo sample to account for the effect of the detection acceptance and efficiency. The $\left|\cos \theta_{H}\right|$ distribution of the "non peaking" background is parameterized by the combination of exponential and constant components.

Figures. 4, 5 and 6 show the $m_{\mathrm{ES}}, \Delta E$ and $\left|\cos \theta_{H}\right|$ distributions for the $K^{+} \pi^{-}, K_{S}^{0} \pi^{+}$and $K^{+} \pi^{0}$ modes, respectively. The $\left|\cos \theta_{H}\right|$ distributions in the signal region (defined as $-0.15<$ $\Delta E<0.10 \mathrm{GeV}, 5.272<m_{\mathrm{ES}}<5.288 \mathrm{GeV} / c^{2}$ ) are also shown. The signal as well as background yields are allowed to vary in the fit. All the non peaking background parameters are allowed to float. The signal and peaking-background helicity angle, Crystal-Ball width and shape parameters 
are constrained according to the $\mathrm{MC}$ expectations. The means of the signal $m_{\mathrm{ES}}$ and $\Delta E$ functions are constrained to the MC expectations, calibrated using $B \rightarrow K^{*}(892) \gamma$ candidates from MC simulation and data, while the peaking-background means are allowed to float due to its complex composition. The signal and peaking-background yields are given in Table 1 . The signal significance has been evaluated from the change in the likelihood when the fit is repeated with the signal yield set to zero.
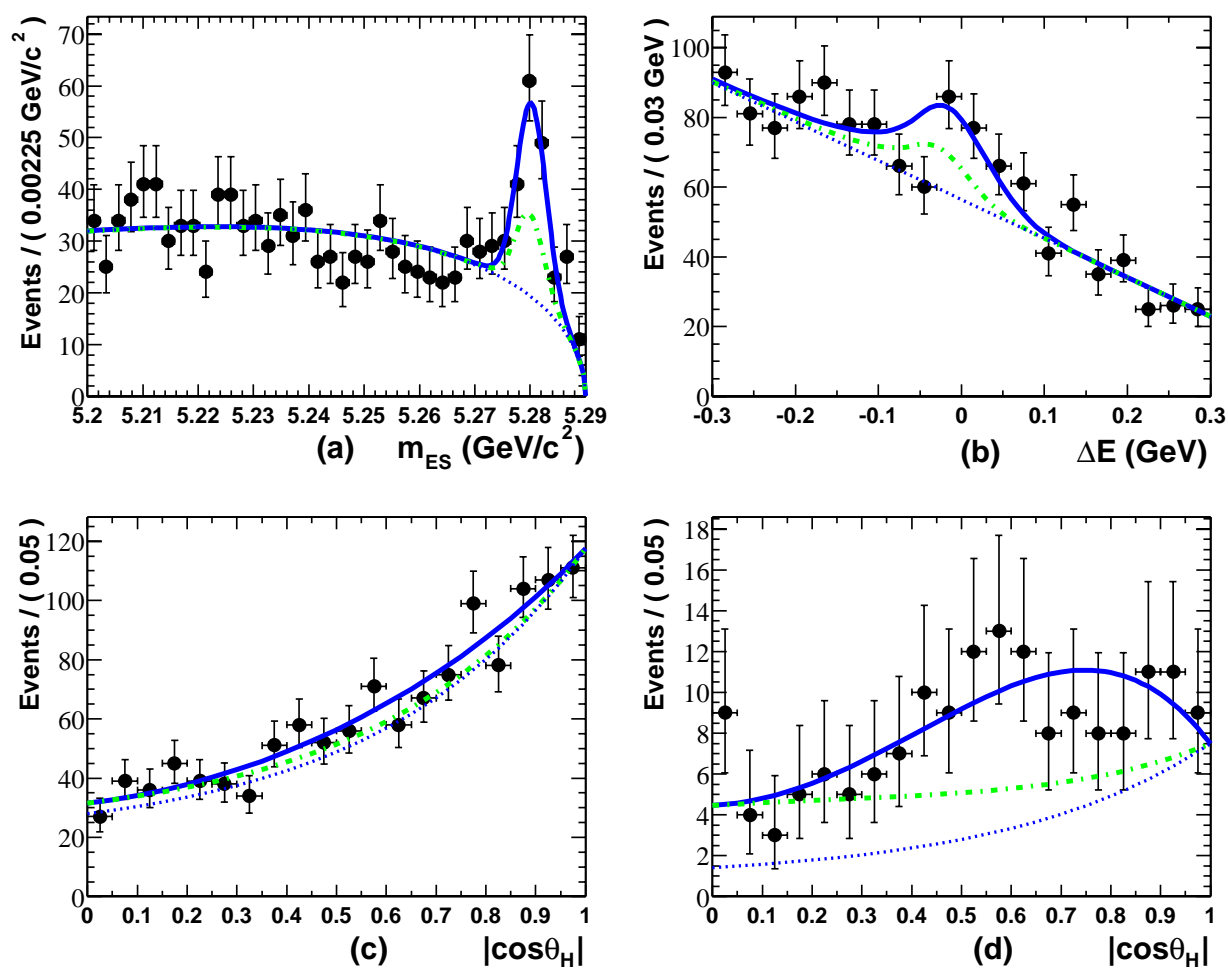

Figure 4: A fit to the (a) $m_{\mathrm{ES}}$, (b) $\Delta E$ and (c) $\left|\cos \theta_{H}\right|$ distributions for $B^{0} \rightarrow K_{2}^{*}(1430)^{0} \gamma$, $K_{2}^{*}(1430)^{0} \rightarrow K^{+} \pi^{-}$candidates in data, and (d) the $\left|\cos \theta_{H}\right|$ distribution in the signal region. The solid line shows a fit to the data. The peaking (dash-dot) and non peaking (dash) background contributions are also shown.

Figure. 7 shows the $K \pi$ invariant mass distribution, which is fit with a relativistic Breit-Wigner function plus a first-order polynomial background, though we understand that it is not the most accurate description of the resonance due to possible interferences. There is a clear enhancement around $1.4 \mathrm{GeV} / c^{2}$ in the neutral mode and a slight enhancement in the charged modes.

\section{SYSTEMATIC STUDIES}

The total systematic error is the sum in quadrature of the components shown in Table 2 . The $\Delta E$ resolution is dominated by the photon energy resolution, which is determined from data using $\pi^{0}$ and $\eta$ meson decays with symmetric decay-photon energies. The deviation in the reconstructed $\eta$ mass from the nominal $\eta$ mass provides an estimate of the uncertainty in the measured single photon 
Table 1: The efficiency, fitted signal yield, peaking-background yield and measured branching fraction $\mathcal{B}\left(B \rightarrow K_{2}^{*}(1430) \gamma\right)$ for each $K_{2}^{*}(1430)$ decay mode.

\begin{tabular}{|c|c|c|c|c|c|}
\hline Mode & $\begin{array}{c}\text { Efficiency } \\
(\%)\end{array}$ & Signal & $\begin{array}{c}\text { Peaking } \\
\text { background }\end{array}$ & $\begin{array}{c}\text { Signal } \\
\text { significance }\end{array}$ & $\begin{array}{c}\mathcal{B}\left(B \rightarrow K_{2}^{*}(1430) \gamma\right) \\
\left(10^{-5}\right)\end{array}$ \\
\hline$K^{+} \pi^{-}$ & 6.4 & $69 . \pm 14$. & $47 . \pm 12$. & 5.8 & $1.22 \pm 0.25 \pm 0.11$ \\
\hline$K_{S}^{0} \pi^{+}$ & 1.9 & $29 . \pm 10$. & $11 . \pm 8$. & 3.3 & $1.69 \pm 0.59 \pm 0.20$ \\
\hline$K^{+} \pi^{0}$ & 1.9 & $20 . \pm 9$. & $4 . \pm 7$. & 2.6 & $1.23 \pm 0.55 \pm 0.12$ \\
\hline
\end{tabular}

energy. The photon isolation and $\pi^{0} / \eta$ veto efficiency depends on the event multiplicity and the effect is estimated by "embedding" MC-generated photons into both an exclusively reconstructed $B$ meson data sample and a generic $B$-meson MC sample. The photon and $\pi^{0}$ efficiency uncertainties are determined from a comparison of the efficiencies in data and MC for $e^{+} e^{-} \rightarrow \tau^{+} \tau^{-}$events. The tracking-efficiency uncertainty is estimated from a sample of tracks well measured in the SVT. We estimate the uncertainties in the $K_{S}^{0}$ efficiency by comparing the data and MC distributions of the momentum and flight distance. The efficiency for kaon identification in the DIRC is derived from a sample of the decays $D^{*+} \rightarrow D^{0} \pi^{+}$, with $D^{0} \rightarrow K^{-} \pi^{+}$.

The uncertainties in the background suppression cuts (the thrust angle and neural network cuts) are obtained by comparing the cut efficiencies between the $B \rightarrow K_{2}^{*}(1430) \gamma \mathrm{MC}$, and $B \rightarrow D \pi^{+}$ data and MC samples, and choosing the largest discrepancy. The invariant mass and width used for $K_{2}^{*}(1430)$ are from the PDG [8]. The systematic error is obtained by varying the mean and width, within their errors, and using that which results in the largest change in efficiency.

We estimate the systematic error due to the fitting procedures as follows. For the shape parameters for $m_{\mathrm{ES}}, \Delta E$ and $\left|\cos \theta_{H}\right|$ distributions, we vary the parameters in the fit within their errors from the MC expectations. We also test the validity of the peaking-background $\left|\cos \theta_{H}\right|$ probability density function (PDF) by replacing it with different parameterized PDFs from MC samples. We double the largest deviation in these tests as the systematic error of the signal yield. There is also a small systematic error associated with the limited statistics of the signal MC sample.

\section{CONCLUSIONS}

We have presented a preliminary measurement of the branching fraction for $B^{0} \rightarrow K_{2}^{*}(1430)^{0} \gamma$ of $(1.22 \pm 0.25 \pm 0.11) \times 10^{-5}$ with a $5.8 \sigma$ statistical significance, which is an improvement over previous experimental results, while still being in agreement. We observe a signal with a statistical significance of $4.1 \sigma$ for $B^{+} \rightarrow K_{2}^{*}(1430)^{+} \gamma$ and measure the preliminary branching fraction to be $(1.44 \pm 0.40 \pm 0.13) \times 10^{-5}$, by combining the results from $K_{S}^{0} \pi^{+}$and $K \pi^{0}$ modes. Both results agree with the theoretical prediction from the relativistic form-factor model of Veseli and Olsson [13].

\section{ACKNOWLEDGMENTS}

We are grateful for the extraordinary contributions of our PEP-II colleagues in achieving the excellent luminosity and machine conditions that have made this work possible. The success of this project also relies critically on the expertise and dedication of the computing organizations that support BABAR. The collaborating institutions wish to thank SLAC for its support and the 

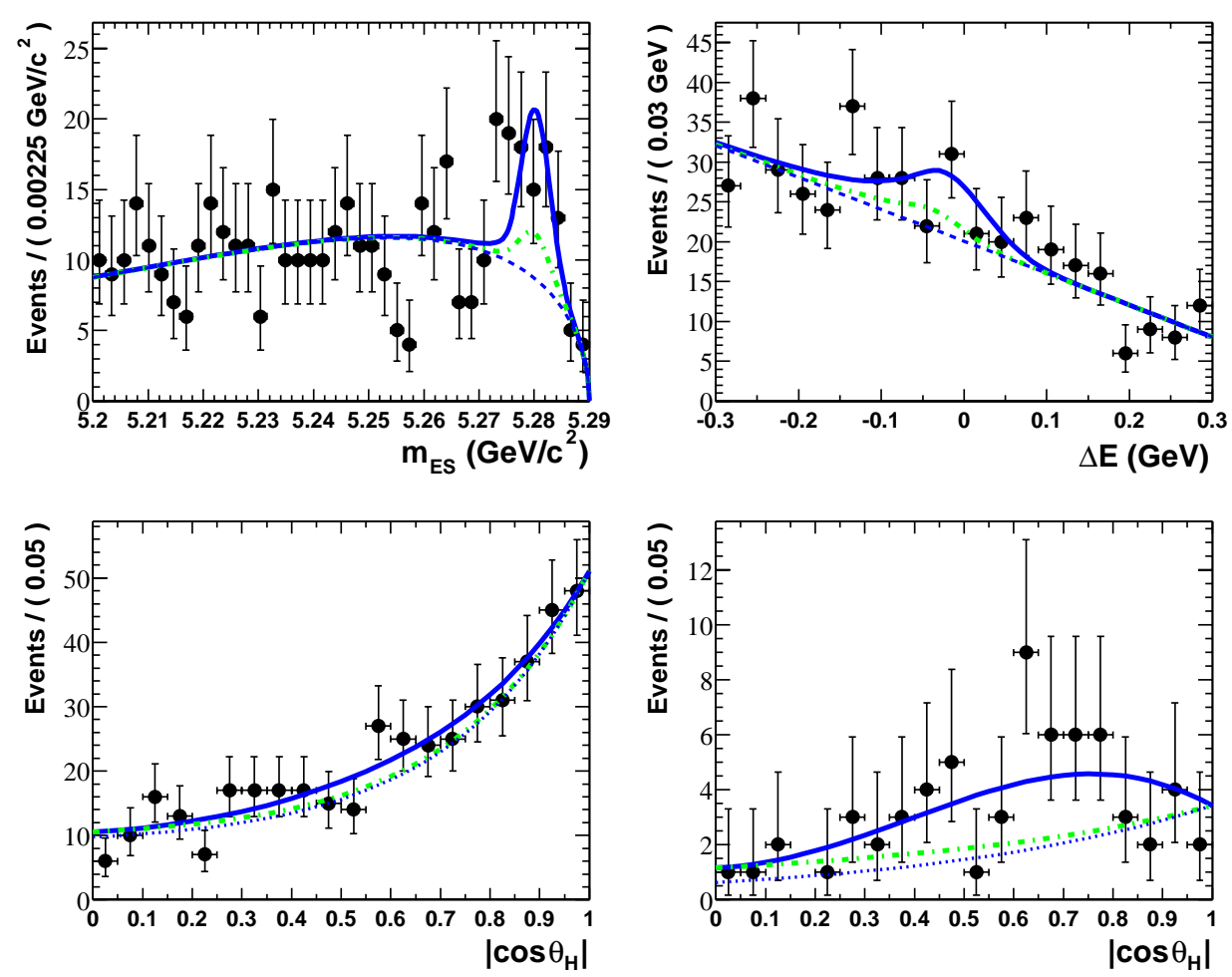

Figure 5: A fit to the (a) $m_{\mathrm{ES}}$, (b) $\Delta E$ and (c) $\left|\cos \theta_{H}\right|$ distributions for $B^{+} \rightarrow K_{2}^{*}(1430)^{+} \gamma$, $K_{2}^{*+}(1430) \rightarrow K_{S}^{0} \pi^{+}$candidates in data, and (d) the $\left|\cos \theta_{H}\right|$ distribution in the signal region. The solid line shows a fit to the data. The peaking (dash-dot) and non peaking (dash) background contributions are also shown.

kind hospitality extended to them. This work is supported by the US Department of Energy and National Science Foundation, the Natural Sciences and Engineering Research Council (Canada), Institute of High Energy Physics (China), the Commissariat à l'Energie Atomique and Institut National de Physique Nucléaire et de Physique des Particules (France), the Bundesministerium für Bildung und Forschung and Deutsche Forschungsgemeinschaft (Germany), the Istituto Nazionale di Fisica Nucleare (Italy), the Foundation for Fundamental Research on Matter (The Netherlands), the Research Council of Norway, the Ministry of Science and Technology of the Russian Federation, and the Particle Physics and Astronomy Research Council (United Kingdom). Individuals have received support from the A. P. Sloan Foundation, the Research Corporation, and the Alexander von Humboldt Foundation.

\section{References}

[1] A.J. Buras et al. , Nucl. Phys. B 631, 219 (2002).

[2] J. Hewett and J. Wells, Phys. Rev. D 55, 5549 (1997).

[3] CLEO Collaboration, T.E. Coan et al. , Phys. Rev. Lett. 84, 5283 (2000). 

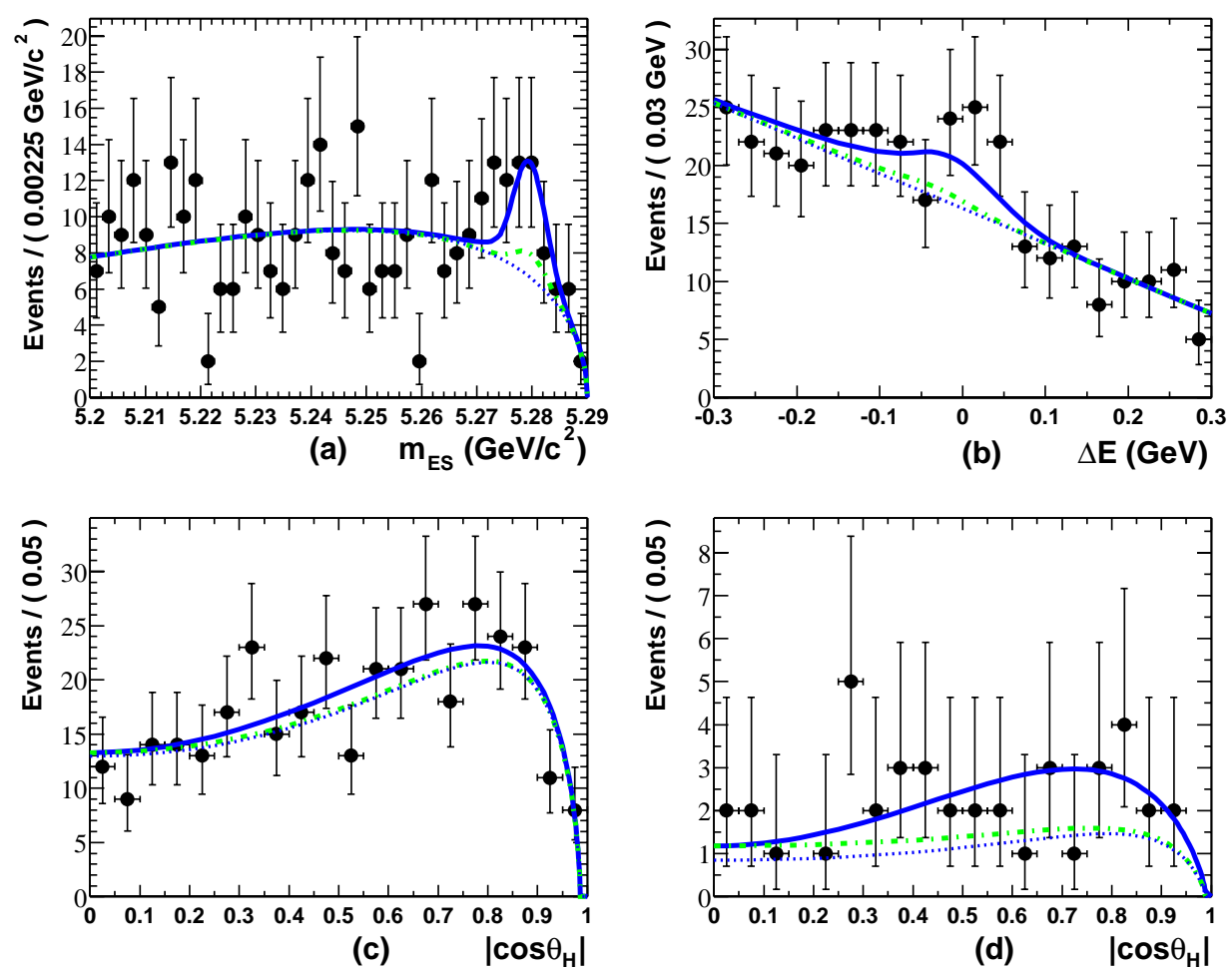

Figure 6: A fit to the (a) $m_{\mathrm{ES}}$, (b) $\Delta E$ and (c) $\left|\cos \theta_{H}\right|$ distributions for $B^{+} \rightarrow$ $K_{2}^{*}(1430)^{+} \gamma, K_{2}^{*+}(1430) \rightarrow K^{+} \pi^{0}$ candidates in data, and (d) the $\left|\cos \theta_{H}\right|$ distribution in the signal region. The solid line shows a fit to the data. The peaking (dash-dot) and non peaking (dash) background contributions are also shown.

[4] BELLE Collaboration, S. Nishida et al. , Phys. Rev. Lett. 89, 231801 (2002).

[5] ALEPH Collaboration, R. Barate et al., Phys. Lett. B 429, 169 (1998);

CLEO Collaboration, D. Cronin-Hennessy et al., Phys. Rev. Lett. 87, 251807 (2001);

BELLE Collaboration, K. Abe et al., Phys. Lett. B 511, 151 (2001);

BABAR Collaboration, B. Aubert et al., hep-ex/0207076 and hep-ex/0207074, Contributed to 31st International Conference on High Energy Physics (ICHEP 2002) .

[6] BABAR Collaboration, B. Aubert et al., Nucl. Instrum. Methods A 479, 1 (2002).

[7] GEANT4 Collaboration, V.N. Ivanchenko et al., Nucl. Instrum. Methods A 494, 514 (2002).

[8] Particle Data Group, K. Hagiwara et al., Phys. Rev. D 66, 010001 (2002).

[9] G.C. Fox and S. Wolfram, Nucl. Phys. B 149, 413 (1979).

[10] http://www-ra.informationkit.uni-tuebingen.de/SNNS/ .

[11] D. Antreasyan, Crystal Ball Note 321 (1983). 

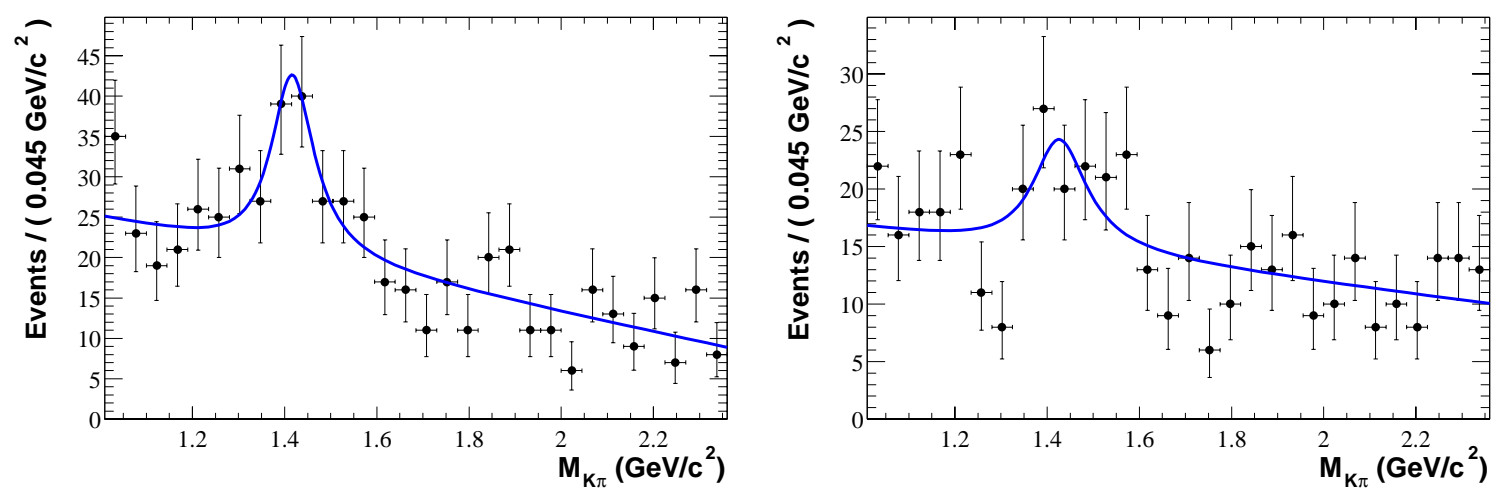

Figure 7: $K^{+} \pi^{-}$(left) and $K_{S}^{0} \pi^{+}$and $K^{+} \pi^{0}$ (right) invariant mass distributions for the signal region (see text for a definition of the signal region).

Table 2: Fractional systematic uncertainties (\%) in the measurement of $\mathcal{B}\left(B \rightarrow K_{2}^{*}(1430) \gamma\right)$.

\begin{tabular}{|l|c|c|c|}
\hline \multirow{2}{*}{ Uncertainty } & \multicolumn{3}{|c|}{} \\
\cline { 2 - 4 } & $K^{ \pm} \pi^{\mp}$ & $K_{S}^{0} \pi^{ \pm}$ & $K^{ \pm} \pi^{0}$ \\
\hline$B$-counting & 1.1 & 1.1 & 1.1 \\
Photon detection efficiency & 1.3 & 1.3 & 1.3 \\
Photon energy scale & 1.0 & 1.0 & 1.0 \\
Photon energy resolution & 2.5 & 2.5 & 2.5 \\
Photon isolation & 2.0 & 2.0 & 2.0 \\
$\pi^{0} / \eta$ veto & 1.0 & 1.0 & 1.0 \\
$K^{+} / \pi^{+}$tracking & 1.6 & 0.8 & 0.8 \\
$K_{S}^{0}$ efficiency & $\ldots$ & 3.0 & $\ldots$ \\
$\pi^{0}$ efficiency & $\ldots$ & $\ldots$ & 2.5 \\
Sub-mode branching fraction & 2.4 & 2.4 & 2.4 \\
$K_{2}^{*}(1430)$ mass/width & 1.6 & 1.0 & 1.1 \\
Signal PDF parameters & 3.9 & 5.8 & 6.3 \\
Background suppression & 5.0 & 6.0 & 6.0 \\
Peaking-background modeling & 3.0 & 5.0 & 5.0 \\
MC statistics & 2.5 & 3.2 & 3.2 \\
\hline Total & 9. & 12. & 10. \\
\hline
\end{tabular}

[12] The background for each mode is fit with $d N / d m_{\mathrm{ES}} \propto \frac{M_{E S}}{E_{\text {beam }}} \times \sqrt{1-\frac{M_{E S}^{2}}{E_{\text {beam }}^{2}}} \times \exp \left(-\zeta\left(1-\frac{M_{E S}{ }^{2}}{E_{\text {beam }}^{2}}\right)\right)$, a function introduced by the ARGUS Collaboration. H. Albrecht et al., Z. Phys. C 48, 543 (1990).

[13] S. Veseli and M.G. Olsson, Phys. Lett. B 367, 309 (1996). 